\title{
Sinkhole collapse propagation studies through instrumented small-scale physical models
}

\author{
Maria Ferentinou \\ Department of Civil Engineering Science, University of Johannesburg, Johannesburg, 2006, South Africa \\ Correspondence: Maria Ferentinou (mferentinou@uj.ac.za)
}

Published: 22 April 2020

\begin{abstract}
Sinkholes are common geohazards, frequently responsible for sudden catastrophic ground collapse. Thus, effective monitoring would allow for further understanding of the mechanism of occurrence of sinkholes and lead to the development of a potential early warning system to provide an alarm or a warning of incipient collapse. In the current study, fiber Bragg gratings (FBGs) were used to instrument reduced scale models, simulating a sinkhole event. The tests were conducted by embedding optic fiber sensors in the soil and inducing failure until critical conditions were reached. FBG sensors were manufactured in a single optic fiber cable. The measurements of small horizontal strains were recorded simultaneously and in various positions. Failure mechanism was found to relate to the backfill density, and compaction.
\end{abstract}

\section{Introduction}

Sinkholes are expressed on the ground surface as surface depressions, due to lack of support of the soil and rock hosting and existing vault. They occur with little or no warning and directly or indirectly affect communities and major and minor infrastructure. There are various events recorded worldwide with hotspots in Florida, South Africa, or the Deep Sea in karst dominated areas. They often initiate as small cavities and expand due the prolonged action of water seeping through the overburden (Fig. 1). Eventually the cavity becomes large enough that the remaining overburden is no longer able to arch across the cavity and collapses (Augarde et al., 2003). Unstable cavities may be formed due mining activities or leaking pipes. The term used in mining is "Chimney caving" for the formation of a sinkhole due to unsupported mined void through the overlying material (Brady and Brown, 1993). The initial sinkhole expression to the surface is usually a small indicator of the final size of the subsequent failure of the overhanging soil around.

Popular methods for high precision recording of displacement in soil span from noncontact laser techniques which measure surface displacement of the soil, point, line or area based (i.e. laser radar, laser line triangulation, shape from shading, close - range photogrammetry and particle image velocimetry (PIV), White and Bolton (2002). Linear variable

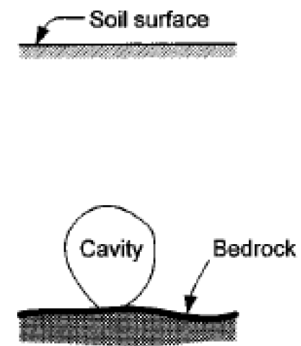

(a)

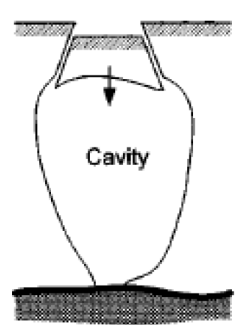

(b)

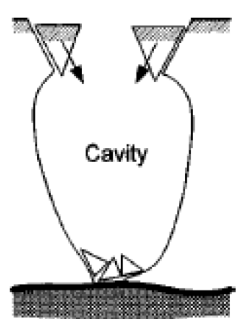

(c)
Figure 1. Conceptual model of cavity propagation (Augarde et al., 2003).

differential transformers (LVDTs) can measure internal deformation and linear displacement within soil (Zhang et al., 2017). There are various types of strain gauges, mechanical, electrical, optical, pneumatic and acoustical strain gauges to name a few, that measure the strain on the surface of a soil. These methods measure displacement at the surface of the soil, but do not measure displacement internally.

Therefore the use of a relatively new technology like fiber optic sensors to monitor the internal deformation for sinkhole or subsidence precursor phenomena detection seems to be promising. 


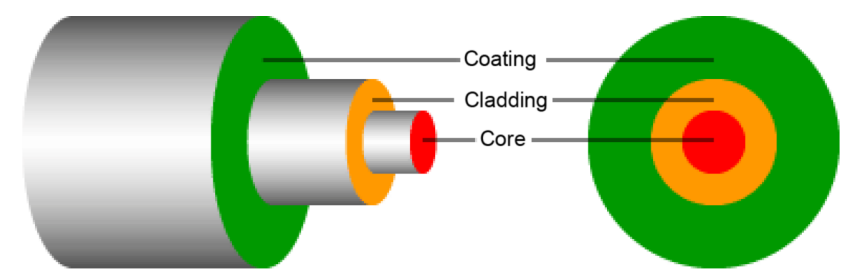

Figure 2. Optic Fiber structure, (C) National Instrument.

In this paper we use reduced scale models and monitor the variation for different experiment conditions, especially in the range of small strain at early stages of failure, can lead to early prediction of horizontal and vertical ground displacement. This kind of technology can inform further on failure mechanism, of sinkhole collapse and the critical factors that influence the response of the prototype through the study of the model.

There is a large amount of previous work on sinkhole propagation studies through physical modelling. The stability of soils was approached through the use of normal gravity tests and geotechnical centrifuge tests in order to examine the stability of cohesive layers in Craig (1990) and weakly cemented layers in Abdula and Goodings (1996), Jacobsz (2016), over circular openings. Costa et al. (2009), performed studies on active trapdoors in granular soil simulating deep and shallow conditions. Among their findings was that the surficial settlement is influenced by the relative density $\left(D_{\mathrm{r}}\right)$ of the soil. In this study we further report on the effect of relative density based on tests on small scale models instrumented by fiber optic sensors under normal gravity conditions.

\section{Introduction to Fiber optic sensing}

\subsection{Principles of fiber optic sensing}

In fiber optic technology, fiber (cables) transmit continuously modulated analogue streams of light, or a series of digital pulses from one point to another along the optic fiber. A side view of typical single-mode optic fiber is shown in Fig. 2. Cladding material with higher refraction index keeps the propagating light pulse inside the glass core. The buffer coating and the jacket are used to offer resistance to external or internal interferences and for the protection of the glass core (Iten, 2011).

The pulses are generated to specific characteristics by an optical spectrum analyser. Once a pulse has propagated through optic fiber, it is a fed into an interrogator or optical spectrum analyser (Othonos, 2000). The pulse is then analysed by the device, to determine any attenuation or change in wavelength (which may have resulted from scattering during the pulse's propagation). The popularity of fiber optic sensing techniques has risen due to several advantages it has over conventional sensing techniques. These are immunity to electromagnetic interference, immunity to power fluctuation along the optical path, insensitivity to corrosion and fatigue, high precision and durability, and reduced size and cable requirement.

\subsection{Fiber Bragg Gratings (FBGs)}

The photosensitivity of optical fibers allows for the formation of phase structures within its' core, called gratings (Othonos, 2000). The operational principles of fiber Bragg gratings (FBGs) is based on the presence of these gratings within the optical fiber which are created as a series of density alterations positioned periodically along the optical fiber glass core (Iten, 2011). The principle of operation is based on Bragg's law.

According to Bragg's law, a portion of light travelling through the optic fiber, with a specific wave-length, is reflected when it passes a Bragg grating. The value of this specific wavelength at which a light ray is reflected, is called Bragg wavelength. This value is dependent on the distribution of the Bragg gratings along the optic fiber (grating period) as well as the refractive index of optic fiber All the other light rays with different wavelengths pass the Bragg grating undisturbed. The light ray that is reflected provides information for potential strain changes, Fig. 3. This is because the Bragg grating period is dependent on the strain in the specimen being monitored (Iten, 2011). The FBG wave-length change is sensitive to tensile and compression stress and temperature. The relationship between the refractive index $n_{\mathrm{e}}$ of the fiber's core, grating period $\Lambda$ and Bragg wavelength $\lambda_{B}$ is expressed mathematically following Eq. (1):

$\lambda_{\mathrm{B}}=2 n_{\mathrm{e}} \Lambda$

where $n_{\mathrm{e}}$ is the effective refractive index of the grating in the fiber core and $\Lambda$ is the grating period. An FBG is sensitive to elongation and temperature, following Eq. (2):

$\frac{\Delta \lambda_{\mathrm{B}}}{\lambda_{\mathrm{B}}}=\left(1-p_{\mathrm{e}}\right) \varepsilon+\left(\alpha_{\Lambda}-a_{n}\right) \Delta T$

Where $\Delta \lambda_{B}$ is the shift of the reflected wavelength $=\left(\lambda_{\mathrm{B}}^{\prime}-\lambda_{\mathrm{B}}\right), \quad p_{\mathrm{e}}$ is the photoelastic coefficient, $a_{\Lambda}$ is the thermal coefficient, and $a_{n}$ is the thermal modulation of the core refractive index. The shift of reflected wavelength has a linear relationship with the axial strain $\varepsilon$ and the change of temperature $\Delta T$. Temperature sensors (FBGs which are covered and protected from strain) are usually embedded to detect temperature variations in the specimen being monitored. Temperature variations are usually not observed for short-term physical-model tests.

\section{Reduced-Scale Physical Model Description}

\subsection{General characteristics}

A simple sinkhole propagation simulation model was designed and developed, to verify the use of optical fiber sens- 


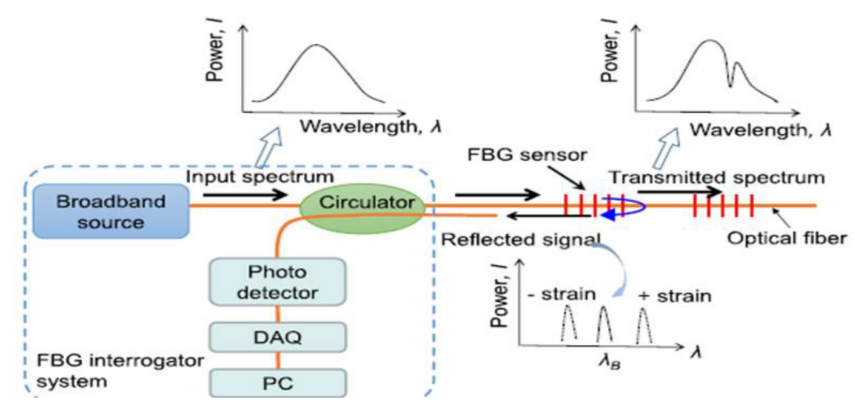

Figure 3. Fiber Bragg grating system, adopted from $\mathrm{Xu}$ et al. (2017).

Table 1. Scaling laws for applicable physical properties.

\begin{tabular}{lr}
\hline Property (prototype) $1 g$ & Scale factor (model) \\
\hline Length & $N$ \\
Mass density & 1 \\
Stress & 1 \\
Displacement & $N$ \\
Strain & 1 \\
\hline
\end{tabular}

ing fibers for deformation measurement. The sinkhole model was constructed inside a Perspex box, with inner dimensions of $400 \times 270 \times 80 \mathrm{~mm}$ (length $\times$ height $\times$ width). The sinkhole was represented through a balloon full of water. The sinkhole collapse was induced by the deflation of a balloon, using a controlled valve. The diameter of the balloon was $90 \mathrm{~mm}$, representing a prototype of $12 \times 8.1 \times 2.4 \mathrm{~m}$, using a scale of $1: 30$. The prototype is representing the scenario of a medium sinkhole $2-5 \mathrm{~m}$, at medium $(1-5 \mathrm{~m})$ to large (5$15 \mathrm{~m}$ ) depth categories (Buttrick et al., 2001). For model tests to be meaningful, similitude must be established between stresses and strains in the model and prototype. The model is reduced by a scale factor $(N)$ scaling laws can be used to convert the measured properties and values of the model to a full-scale prototype equivalent. Applicable scaling laws are shown in Table 1.

The layers of sand were placed and four optic fibre cables with FBGs on each optic fibre cable were placed. Twelve strain sensors (S1-S12) were multiplexed into 3 FBGs per fibre cable, $53 \mathrm{~mm}$ apart, to determine the maximum expected strain and strain near the boundaries of the cavity as it propagates upwards, Fig. 4. The FBG sensors were manufactured in single mode photosensitive fibre in the Photonics Research laboratory of the University of Johannesburg using the phase mask technique and an Nd:YAG laser using the $266 \mathrm{~nm}$ wavelength. The FBG sensors were printed to reflect in the wavelength range between 1540 and $1555 \mathrm{~nm}$.

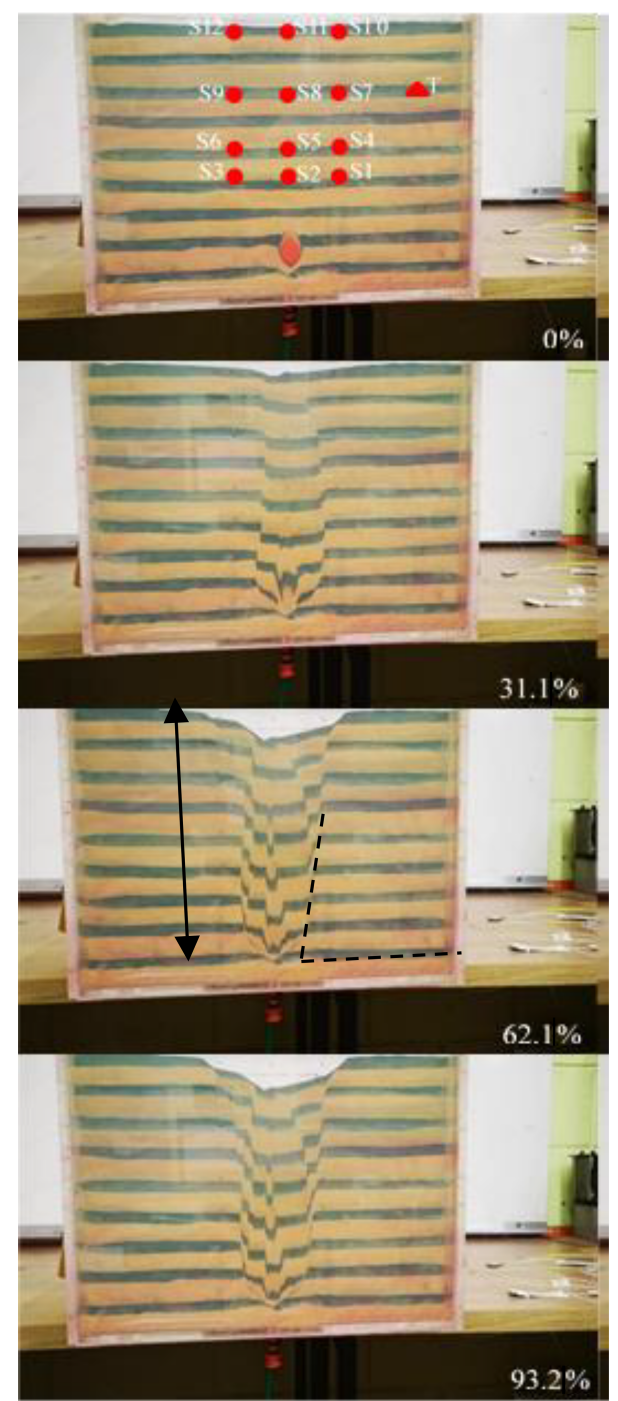

Figure 4. Failure surfaces with medium compact sand fill, the percentages refer to balloon volume reduction (photo taken by Jeandre Labuschagne).

\subsection{Material properties}

The soil used for the experimental work was a silica sand known as Cullinan sand from a commercial site. The poorly graded sand was characterised as SP, and had a D50 particle diameter of $0.15 \mathrm{~mm}$. The sand angle of repose ws measured to be $37^{\circ}$. Coloured sand was used as an indicator to enable simultaneously record and display internal deformation process. The sand was placed and compacted in alternating layers of uncoloured sand $(20 \mathrm{~mm})$ and coloured sand $(10 \mathrm{~mm})$.

\subsection{Testing program}

The experimental component of this project included three tests of reduced scaled models. The variable that was investigated was sand relative density $D_{\mathrm{r}}$. The models we rebuild to 


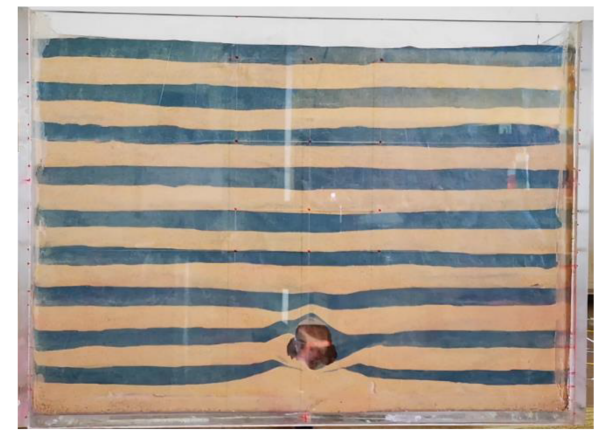

Figure 5. Very well compacted sand fill, at a $100 \%$ volume reduction (photo taken by Jeandre Labuschagne).

sand relative density values of $60 \%, 65 \%$ and $95 \%$ and densities of $1560.98,1623.41,1705.61 \mathrm{~kg} \mathrm{~m}^{-3}$ respectively and were tested under normal gravity $(1 g)$. The water-inflated balloon had a diameter of $90 \mathrm{~mm}$. The deflation of the balloon allowed for the upwards propagation of the sinkhole in two-dimensional plane-strain conditions, Jacobsz (2016). The balloon was deflated by letting water out at a flow rate of $34.17,47.40$ and $41.43 \mathrm{~mL} \mathrm{~min}^{-1}$ respectively. The induced movement due to the balloon deflation was $90 \mathrm{~mm}$, which allowed for the visualisation of fully developed failure surfaces within the soil mass.

\section{Results}

\subsection{Failure mechanism}

Figures 4, 5 and 6 show the failure surface that developed as was observed from the Plexiglass wall for the $60 \%$ (test 1 ) and $65 \%$ (test 2) and $95 \%$ (test 3 ) relative density of the sand. The parameters that were used in order to describe the failure surface, are the angle with horizontal $\left(\theta_{i}\right)$, and the maximum height of the failure surface measured from the base of the model $\left(h_{v}\right)$ (Costa et al., 2009).

According to the results in both tests a failure surface on both sides of the balloon initiating at $6 \mathrm{~cm}$ from the centre developed towards the middle of the model, at $10 \%$ volume reduction and eventually propagated to the surface at $22 \%$ reduction volume. The observed failure pattern is that of a chimney caving with a width of 3.80 and $2.28 \mathrm{~cm}$ for test 1 and test 2 respectively. Secondary failure surfaces developped after $30 \%$ volume reduction from the middle of the height to the surface with an angle of $80^{\circ}$ to the horizontal. The maximum settlement was $2.80 \mathrm{~cm}$ for test 1 and $2.28 \mathrm{~cm}$ for test 2. The settlement trough width was 11.4 and $14.28 \mathrm{~cm}$ for test 1 and test 2 respectively. The chimney width was 3.8 and $5.71 \mathrm{~cm}$ for test 1 and test 2 respectively.

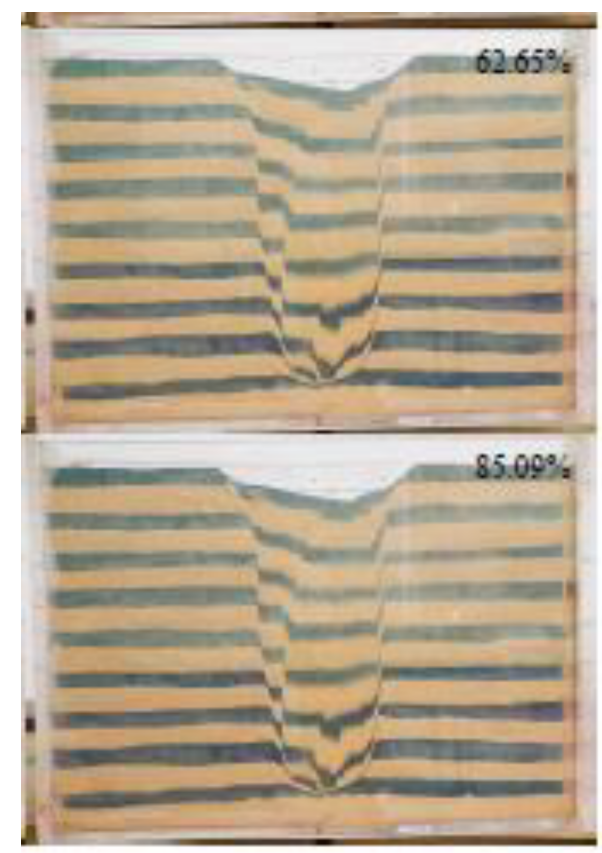

Figure 6. Failure surfaces with compact sand fill, the percentages refer to balloon volume reduction (photo taken by Esmerelda Steyn).

\subsection{FBGs Measurement results}

The analysis of the experimental results that were recorded by the FBGs sensors were used in order to identify a subsidence pattern above the balloon resulting from the induced collapse. Each sensor array contained three gratings that were written along a single fiber by using UV laser. The sensing length of each FBG was $6 \mathrm{~mm}$. The spacing between FBGs in one fiber was specified for the collection of strain at certain positions. Fiber 1, 2, 3, 4 were installed horizontally (Fig. 4).

Figures 7 and 8 present the strain variation of the sensors for the two models. The results from test 1 showed that

the maximum strain was measured by sensor $\mathrm{S} 4$ approximately $7.5 \times 10^{-3}$ in $n \varepsilon$ at $40 \%$ balloon volume reduction. $\mathrm{S} 1$ in fiber 1 close to the left side of the balloon measured $8 \times 10^{-4} n \varepsilon$. S7, S8, S9 measured $6 \times 10^{-4} n \varepsilon$ between $40 \%$ and $80 \%$ volume reduction. Interestingly S10, S11, S12, did not measure high strains as it would theoretically has been expected based on settlement experienced.

The results from test 2 indicated that the maximum strain was measured by sensor S3 approximately $6 \times 10^{-4} n \varepsilon$ at $30 \%$ balloon volume. S6 measured $7 \times 10^{-4}$ and S9 $1 \times 10^{-3}$. The recorded strain relates to the secondary failures. The sensors located at the centre and the array laid at the highest level within the model measured very low strain which does not verify what would be expected from the empirical observation of surficial settlements induced by excavations of tunnels (Peck, 1969). 

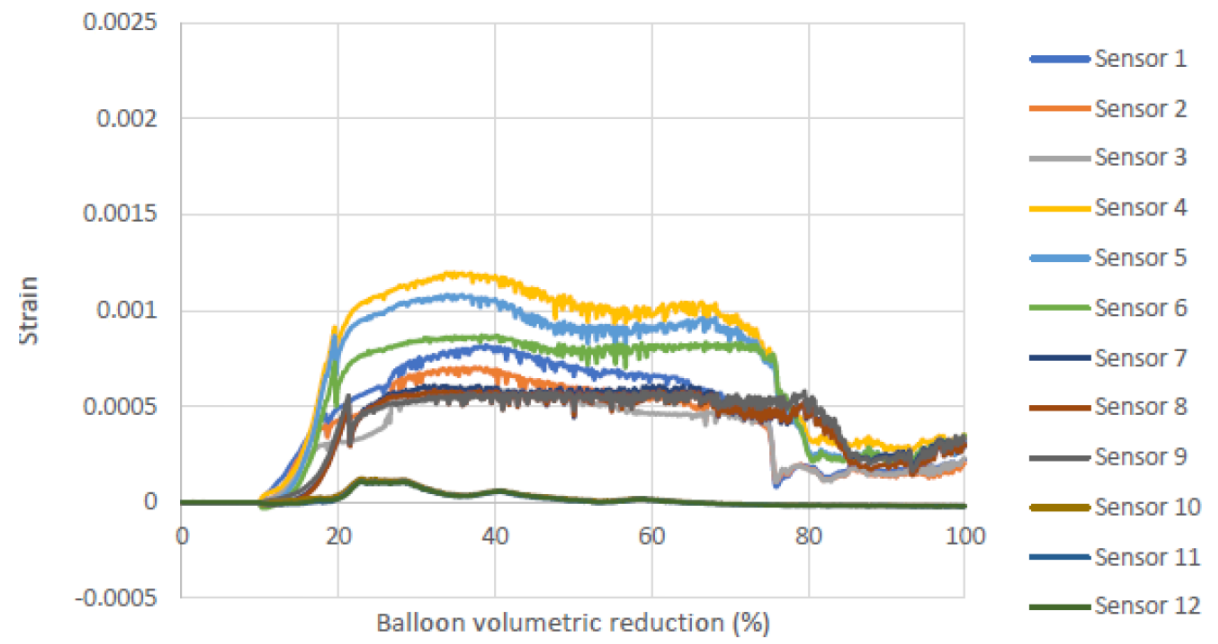

Figure 7. Strain variation for of all FBGs during test 1.
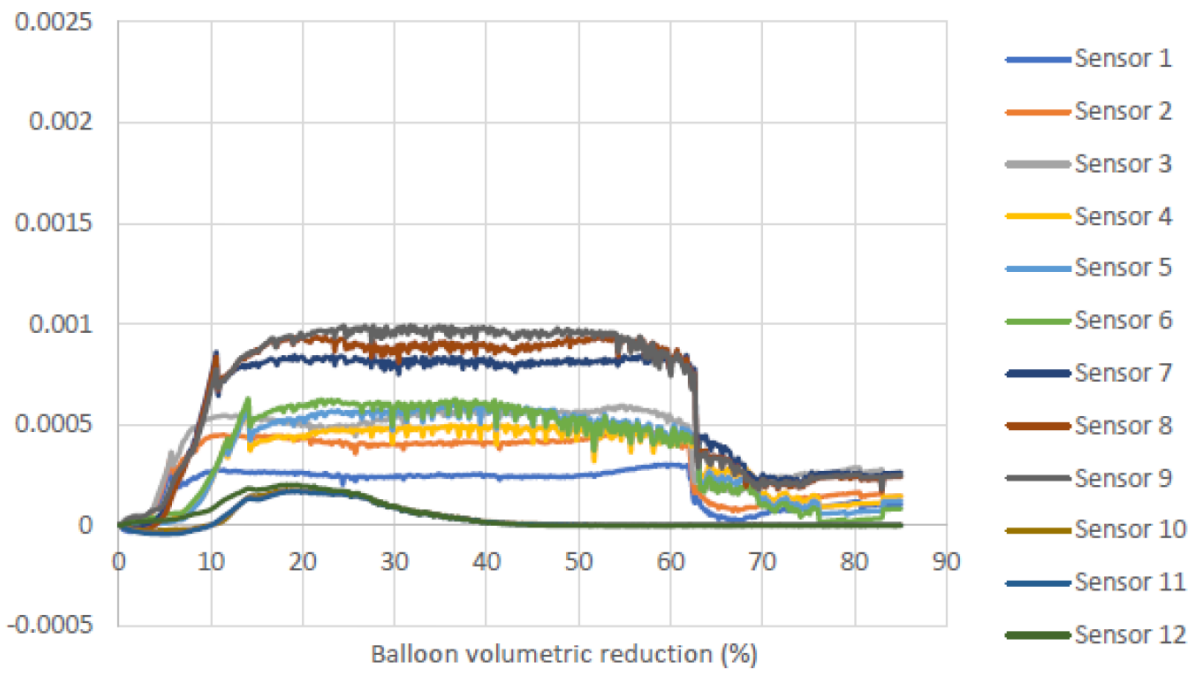

Figure 8. Strain variation for of all FBGs during test 2.

\section{Conclusions}

The failure mechanism in a granular soil induced by the deflation of a balloon simulating an underground cavity was studied in this paper, though small-scale models. The models were built with a ratio of overburden $(H)$ to the balloon diameter $(D)$ of 4 , simulating deep conditions. The following conclusions can be drawn:

The mechanism involved an initial well defined vertical failure surface propagating to the surface following a chimney caving pattern, with an evident curvature upwards. A secondary failure surface at $80^{\circ}$ to the horizontal in the region above the balloon develops at almost $35 \%$ volume reduction.

The soil density influenced the magnitude of the surficial settlement, which was almost $2.5 \%$ of the balloon diameter. Surficial settlement was larger in less compacted sands and did not develop at all in very well compacted sands. The max strains that were recorded by FBGs sensors indicated that the highest stains were recorded at the secondary failure surfaces that were developed starting from the sides of the balloon. A complete program of experiments is planned to further investigate the effect of relative density, and water content, in a geotechnical centrifuge facility.

The technique is also planned to be applied at identified pilot areas in collaboration with the local municipalities. Sites that are prone to sinkhole hazard for the last thirty years or so, are going to serve as monitoring sites, where the current methodoly can be fyrther implemented and tested as to providing an early warning system.

Data availability. Data acquired during the project will be available through University of Johannesburg repository. 
Competing interests. The author declares that there is no conflict of interest.

Special issue statement. This article is part of the special issue "TISOLS: the Tenth International Symposium On Land Subsidence - living with subsidence". It is a result of the Tenth International Symposium on Land Subsidence, Delft, the Netherlands, 17-21 May 2021.

Acknowledgements. The author would like to acknowledge, Jeandre Labuschagne and Esmerelda Steyn, for conducting the experiments as part of their research projects, at the University of Johannesburg.

Financial support. This research has been supported by the Water Research Commission in South Africa (grant no. K5 2937) and the National Research Foundation (NRF) (grant no. 113371).

\section{References}

Abdulla, W. A. and Goodings, D. J.: Modeling of sinkholes in weakly cemented sand, J. Geotech. Eng., 122, 998-1005, 1996.

Augarde, C. E., Lyamin, V. A., and Sloan, S.: Prediction of undrained sinkhole collapse, J. Geotech. Geoenviron., 129, 197205, 2003.

Brady, B. H. and Brown, E. T.: Rock Mechanics for underground mining, Chaoman and Hall, London, 1993.

Buttrick, D. B., van Schalkwyk, A. R., Kleywegt, R. J., and Watermeyer, R. B.: Proposed method for dolomite land hazard and risk assessment in South Africa, J. S. Afr. Inst. Civ. Eng., 43, 27-36, 2001.
Costa, Y. D., Zornberg, J. G., Bueno, B. S., and Costa, C. L.: Failure Mechanisms in Sand over a Deep Active Trapdoor, J. Geotech. Geoenviron., 135, 1741-1753, 2009.

Craig, W. H.: Collapse of cohesive overburden following removal of support, Can. Geotech. J., 27, 355-364, 1990.

Iten, M.: Novel Applications of Distributed Fiber Optic Sensing in Geotechnical Engineering, Doctoral thesis, DISS. ETH No. 19632, ETH Zurich, Zurich, 1-33, 2011.

Jacobsz, S. W.: Trapdoor experiments studying cavity propagation, in: Proceedings of the first Southern African Geotechnical Conference, 5-6 May 2016, Suncity, Taylor \& Francis Group, 2016.

Othonos, A.: Bragg Gratings in Optical Fibres: Fundamentals and Applications, Optical Fibre Sensor Technology, 79-187, Kulwar Academic Publishers, the Netherlands, 2000.

Peck, R. B.: State of the art report: Deep excavations and tunnelling in soft ground, Proc. 7th Int. Conf in Soil Mechanics and Foundation Engineering, Mexico City, 225-290, 1969.

White, D. J. and Bolton, M. D.: Soil deformation around a displacement pile in sand, Physical modelling in geotechnics: ICPMG'02, Newfoundland, 649-654, 2002.

Xu, D.-S., Dong, L.-J., Borana, L., and Liu, H.-B.: Early-Warning System With Quasi-Distributed Fiber Optic Sensor Networks and Cloud Computing for Soil Slopes, v5, IEEE Access, 5, 25437 25444, https://doi.org/10.1109/ACCESS.2017.2771494, 2017.

Zhang, D., Xu, Q., Bezuijen, A., Zheng, G., and Wang, H.: Internal deformation monitoring for centrifuge slope model with embedded FBG arrays, Landslides, 14, 407, https://doi.org/10.1007/s10346-016-0742-2, 2017. 